\title{
@creative
}

ISSN 1855-3966 (printed edn.), ISSN 1855-3974 (electronic edn.)

\author{
ARS MATHEMATICA CONTEMPORANEA 17 (2019) 447-454 \\ https://doi.org/10.26493/1855-3974.1917.ea5 \\ (Also available at http://amc-journal.eu)
}

\section{The complement of a subspace in a classical polar space}

\author{
Krzysztof Petelczyc, Mariusz Żynel \\ Institute of Mathematics, University of Biatystok, \\ Ciołkowskiego 1 M, 15-245 Biatystok, Poland
}

Received 22 January 2019, accepted 6 July 2019, published online 7 November 2019

\begin{abstract}
In a polar space, embeddable into a projective space, we fix a subspace, that is contained in some hyperplane. The complement of that subspace resembles a slit space or a semiaffine space. We prove that under some assumptions the ambient polar space can be recovered in this complement.
\end{abstract}

Keywords: Polar space, projective space, semiaffine space, slit space, complement.

Math. Subj. Class.: 51A15, 51A45

\section{Introduction}

Cohen and Shult coined the term affine polar space in [4] as a polar space with some hyperplane removed. They prove that from such an affine reduct the ambient polar space can be recovered. In [9] we prove something similar for the complement of a subset in a projective space. Looking at the results of these two papers it is seen that an interesting case has been set aside: the complement of a subspace in a polar space. We are trying to fill this this gap here, although under several specific assumptions: we consider classical polar spaces, i.e. embeddable into projective spaces (cf. [2]), and our subspace is contained in a hyperplane.

A projective space with some subspace removed is called a slit space (cf. $[5,6,8]$ ) so, our complement can be seen as a generalized slit space. Singular subspaces in a polar space are projective spaces, in an affine polar space they are affine spaces (cf. [4]), while in our complement they are semiaffine or projective spaces. Adopting the terminology of [7], where the class of semiaffine spaces includes affine spaces, projective spaces and everything in between, we could say that singular subspaces of our complement are simply

E-mail addresses: kryzpet@math.uwb.edu.pl (Krzysztof Petelczyc), mariusz@math.uwb.edu.pl (Mariusz Żynel)

(c)(7) This work is licensed under https://creativecommons.org/licenses/by/4.0/ 
semiaffine spaces. This let us call our complement a semiaffine polar space. Anyway, it is clear that the complement we examine is affine in spirit. A natural parallelism is there and the subspace we remove can be viewed as the horizon.

As this paper is closely related to [4] and [9], it borrows some concepts, notations and reasonings from these two works. There are however new difficulties in this case. In contrast to [4], the horizon is not a hyperplane and thus, it induces a partial parallelism (cf. [8]). There are lines disjoint with the horizon in the ambient space and those lines, called non-affine, cannot be parallel to any line in the complement. If we had applied the definition of parallelism from [4] as it is, we would end up with non-affine lines in its equivalence classes. Therefore we use the Veblen condition to express parallelism in terms of incidence in the complement. This method unfortunately is viable only if we have at least 4 points per line in the polar space.

Roughly speaking, the points of the horizon are identified with equivalence classes of parallelism, or, in other words, with directions of lines. On the horizon of an affine polar space a deep point emerges as the point which could be reached by no line of the complement. If the removed subspace is not a hyperplane then there is no deep point but a new problem arises. Some lines on the horizon are recoverable in a standard way, as directions of planes. For the others there are no planes in the complement that would reach them. An analogy to a deep point is clear, so we call them deep lines. To overcome the problem we introduce the following relation: a line $K$ is anti-euclidean to a line $L$ iff there is no line intersecting $K$ that is parallel to $L$. Based on this relation is a ternary collinearity of points on deep lines.

We do not know whether every subspace of a polar space is contained in a hyperplane. Any subspace can be extended to a maximal one, but does it have to be a hyperplane? If that is the case our assumptions could be weakened significantly.

\section{Generalities}

A point-line structure $\mathfrak{M}=\langle S, \mathcal{L}\rangle$, where the elements of $S$ are called points, the elements of $\mathcal{L}$ are called lines, and where $\mathcal{L} \subset 2^{S}$, is said to be a partial linear space, or a point-line space, if two distinct lines share at most one point and every line is of size (cardinality) at least 2 (cf. [3]). A line of size 3 or more will be called thick. If all lines in $\mathfrak{M}$ are thick then $\mathfrak{M}$ is thick. $\mathfrak{M}$ is said to be nondegenerate if no point is collinear with all others, and it is called singular if any two of its points are collinear. It is called Veblenian iff for any two distinct lines $L_{1}, L_{2}$ through a point $p$ and any two distinct lines $K_{1}, K_{2}$ not through the point $p$ whenever each of $L_{1}, L_{2}$ intersects both of $K_{1}, K_{2}$, then $K_{1}$ intersects $K_{2}$. A subspace of $\mathfrak{M}$ is a subset $X \subseteq S$ that contains every line, which meets $X$ in at least two points. A proper subspace of $\mathfrak{M}$ that shares a point with every line is said to be a hyperplane. If $\mathfrak{M}$ satisfies exchange axiom, then a plane of $\mathfrak{M}$ is a singular subspace of dimension 2. A partial linear space satisfying one-or-all axiom, that is

for every line $L$ and a point $a \notin L, a$ is collinear with one or all points on $L$,

will be called a polar space. The rank of a polar space is the maximal number $n$ for which there is a chain of singular subspaces $\emptyset \neq X_{1} \subset X_{2} \subset \cdots \subset X_{n}$ ( $n=-1$ if this chain is reduced to the empty set). For $a \in S$ by $a^{\perp}$ we denote the set of all points collinear with $a$, and for $X \subseteq S$ we put

$$
X^{\perp}=\bigcap\left\{a^{\perp}: a \in X\right\}, \quad \operatorname{rad} X=X \cap X^{\perp} .
$$


As an immediate consequence of one-or-all axiom we get (cf. [4]):

Fact 2.1. For any point $a \in S$ the set $a^{\perp}$ is a hyperplane of $\mathfrak{P}$.

Following [10], a subset $X$ of $S$ is called

- spiky when every point $a \in X$ is collinear with some point $b \notin X$,

- flappy when for every line $L \subseteq X$ there is a point $a \notin X$ such that $L \subseteq a^{\perp}$.

\subsection{Complement}

Let $\mathfrak{M}=\langle S, \mathcal{L}\rangle$ be a thick partial linear space and let $\mathcal{W}$ be a proper subspace of $\mathfrak{M}$. By the complement of $\mathcal{W}$ in $\mathfrak{M}$ we mean the structure

$$
\mathfrak{D}_{\mathfrak{M}}(\mathcal{W}):=\left\langle S_{\mathcal{W}}, \mathcal{L}_{\mathcal{W}}\right\rangle
$$

where

$$
S_{\mathcal{W}}:=S \backslash \mathcal{W} \quad \text { and } \quad \mathcal{L}_{\mathcal{W}}:=\left\{k \cap S_{\mathcal{W}}: k \in \mathcal{L} \wedge k \nsubseteq \mathcal{W}\right\}
$$

The subspace $\mathcal{W}$ will be called the horizon of $\mathfrak{D}_{\mathfrak{M}}(\mathcal{W})$. Note that the complement $\mathfrak{D}_{\mathfrak{M}}(\mathcal{W})$ is a partial linear space. Following a standard convention we call the points and lines of the complement $\mathfrak{D}_{\mathfrak{M}}(\mathcal{W})$ proper, and points and lines of $\mathcal{W}$ are said to be improper. Given a subspace $X$ of $\mathfrak{D}_{\mathfrak{M}}(\mathcal{W})$ its closure $\bar{X}$ is a subspace of $\mathfrak{M}$ with $X \subseteq \bar{X}$. We say that two lines $K, L \in \mathcal{L}_{\mathcal{W}}$ are parallel, and we write

$$
K \|_{\mathcal{W}} L \quad \text { iff } \quad \bar{K} \cap \bar{L} \cap \mathcal{W} \neq \emptyset
$$

This is always an equivalence relation. Its domain is $\mathcal{L}_{\mathcal{W}}$ only in case $\mathcal{W}$ is a hyperplane, or in other words, a line $L \in \mathcal{L}_{\mathcal{W}}$ with $L=\bar{L}$ cannot be parallel to any line. A line $L \in \mathcal{L}_{\mathcal{W}}$ with the property that $L \| \mathcal{W} L$ will be called an affine line. The set of all affine lines, the domain of $\|_{\mathcal{W}}$, will be denoted by $\mathcal{L}^{*}$. For affine line $L$ we write $L^{\infty}$ for the point of $\bar{L}$ in $\mathcal{W}$, i.e. the point at infinity. A point $a \in \mathcal{W}$ is said to be a deep point if there is no line $L \in \mathcal{L}_{\mathcal{W}}$ such that $a=L^{\infty}$. A plane of $\mathfrak{D}_{\mathfrak{M}}(\mathcal{W})$ containing an affine line is said to be a semiaffine plane. By $\Pi^{\infty}$ we denote the set of points at infinity of a semiaffine plane $\Pi$, i.e. $\Pi^{\infty}=\left\{M^{\infty}: M \in \mathcal{L}^{*}\right.$ and $\left.M \subseteq \Pi\right\}$. Note that $\Pi^{\infty}$ is a line iff $\Pi$ is an affine plane. A line $L \subseteq \mathcal{W}$ is said to be a deep line if there is no plane in $\mathfrak{D}_{\mathfrak{P}}(\mathcal{W})$ with $L=\Pi^{\infty}$.

\section{Complement in a polar space}

Let $\mathfrak{P}=\langle S, \mathcal{L}\rangle$ be a thick, nondegenerate polar space of rank at least 3 . In the remainder of the paper we deal with $\mathfrak{D}_{\mathfrak{P}}(\mathcal{W})$, where $\mathcal{W}$ is a proper subspace contained in some hyperplane of $\mathfrak{P}$. Let us emphasize, that we do not mean one particular hyperplane and it is not fixed in our reasonings in any way. If there was a unique hyperplane $H$ containing $\mathcal{W}$ we would be able to recover the ambient space applying Proposition 2.7 from [4], which says that every automorphism of the complement $\mathfrak{D}_{\mathfrak{P}}(H)$ can be uniquely extended to an automorphism of $\mathfrak{P}$. It is not however doable as there could be many hyperplanes containing $\mathcal{W}$ and none of them can be distinguished in terms of the complement.

In polar spaces deep points appear only on hyperplanes and there could be at most one deep point on a hyperplane. 


\section{Lemma 3.1.}

(i) If $\mathcal{W}$ is a hyperplane, then there is at most one deep point on $\mathcal{W}$ and it is in $\operatorname{rad} \mathcal{W}$.

(ii) If $\mathcal{W}$ is not a hyperplane, then there are no deep points on $\mathcal{W}$, that is $\mathcal{W}$ is spiky.

Proof. (i): By Corollary 1.3 (ii) in [4].

(ii): Assume that $a$ is a deep point in $\mathcal{W}$. Then $a^{\perp} \subseteq \mathcal{W}$, and by Fact 2.1 we get that $\mathcal{W}$ contains a hyperplane. A contradiction, as a hyperplane in $\mathfrak{P}$ is a maximal proper subspace (cf. [4, Lemma 1.1]).

Lemma 3.2. Let $\mathfrak{P}$ be an embeddable polar space and $K, L \in \mathcal{L}_{\mathcal{W}}$ be two distinct lines such that $K \|_{\mathcal{W}} L$. The subspace $\mathcal{W}$ can be extended to a hyperplane of $\mathfrak{P}$ not containing $\bar{K}$ and $\bar{L}$.

Proof. If $\mathcal{W}$ is a hyperplane of $\mathfrak{P}$ then $\mathcal{W}$ itself is the required hyperplane.

Assume that $\mathcal{W}$ is not a hyperplane. Let $H$ be a hyperplane containing $\mathcal{W}, \mathfrak{N}$ be a projective space embracing $\mathfrak{P}$, and $f$ be an embedding of $\mathfrak{P}$ into $\mathfrak{N}$. Consider the projective subspace $G$ spanned by $f(H)$. By Proposition 5.2 from [4] $G$ is a hyperplane of $\mathfrak{N}$. If $f(\bar{K}), f(\bar{L}) \nsubseteq G$ then the hyperplane $H=f^{-1}(G \cap f(S))$ is the required one. Let $\mathcal{H}$ be the family of all hyperplanes in $G$ containing $f(\mathcal{W})$.

Now, assume that $f(\bar{K}) \subseteq G$ and $f(\bar{L}) \nsubseteq G$. Take $a_{K} \in f(\bar{K}) \backslash f(\mathcal{W})$ and $a_{L} \in$ $f(\bar{L}) \backslash f(\mathcal{W})$ and choose a hyperplane $G_{0} \in \mathcal{H}$ with $a_{K} \notin G_{0}$. Note that $\overline{a_{K}, a_{L}}$ meets $G$ in $a_{K}$. Take $b \in \overline{a_{K}, a_{L}}$ distinct from $a_{K}$ and $a_{L}$. Assume that there is a line through $b$ that intersects $f(\bar{L}) \backslash f(\mathcal{W})$ in some point $c$ and meets $G_{0}$ in a point $d$. Note that $d \notin f(\bar{K})$ as otherwise we would have $a_{K} \in G_{0}$. Lines $\overline{a_{L}, d}$ and $f(\bar{K})$ are on a plane spanned by the triangle $a_{L}, b, c$. Therefore the line $\overline{a_{L}, d}$ intersects $f(\bar{K})$ in some point $e$ distinct from $d$. Then $\overline{d, e} \subseteq G$, and thus $a_{L} \in G$, a contradiction. Hence, $G^{\prime}=\left\langle G_{0}, b\right\rangle$ is a hyperplane of $\mathfrak{N}$ such that $f(\bar{L}) \nsubseteq G^{\prime}$. We have also $\overline{a_{K}, b} \cap G_{0}=\emptyset$ since $\overline{a_{K}, b} \nsubseteq G$. Thus $f(\bar{K}) \nsubseteq G^{\prime}$. Finally, $H^{\prime}:=f^{-1}\left(G^{\prime} \cap f(S)\right)$ is the hyperplane we are looking for. The case with $f(\bar{K}) \nsubseteq G$ and $f(\bar{L}) \subseteq G$ goes the same way.

Now, let $f(\bar{K}) \subseteq G$ and $f(\bar{L}) \subseteq G$. As in the previous case we take $a_{K} \in f(\bar{K}) \backslash$ $f(\mathcal{W}), a_{L} \in f(\bar{L}) \backslash f(\mathcal{W})$, but this time choose a hyperplane $G_{0} \in \mathcal{H}$ with $a_{K}, a_{L} \notin G_{0}$. Let $b \notin G$. Note that $\overline{a_{i}, b} \cap G_{0}=\emptyset$ for $i=K, L$. So, if we set $G^{\prime}=\left\langle G_{0}, b\right\rangle$ then $f(\bar{K}) \nsubseteq$ $G^{\prime}$ and $f(\bar{L}) \nsubseteq G^{\prime}$. Moreover, $G^{\prime}$ is a hyperplane of $\mathfrak{N}$. Again, $H^{\prime}:=f^{-1}\left(G^{\prime} \cap f(S)\right)$ is the required hyperplane.

Lemma 3.3. Let $K, L \in \mathcal{L}_{\mathcal{W}}$ be two distinct lines such that $K \|_{\mathcal{W}}$ L. There is a sequence $\Pi_{1}, \ldots, \Pi_{n}$ of planes in $\mathfrak{D}_{\mathfrak{P}}(\mathcal{W})$ such that $K^{\infty}=L^{\infty} \in \overline{\Pi_{i}}$ for $i=1, \ldots, n$ and $K \subseteq$ $\Pi_{1}, L \subseteq \Pi_{n}$, and $\Pi_{j}, \Pi_{j+1}$ share a line for $j=1, \ldots, n-1$.

Proof. By Lemma 3.2 we can extend $\mathcal{W}$ to a hyperplane $H$ of $\mathfrak{P}$ such that $K, L \nsubseteq H$. Take the point $a=K^{\infty}$. By (2.1) we have $a=L^{\infty}$. Now, take in $\mathfrak{P}$ the bundle of all the lines together with all the planes through $a$. This structure is actually the quotient space $a^{\perp} / a$, and it is, up to an isomorphism, a polar space (cf. [1, Lemma 2.1]), that we denote by $\mathfrak{P}^{\prime}$. The set $H^{\prime}$, consisting of all the lines through $a$ contained in $H$, is a hyperplane in $\mathfrak{P}^{\prime}$ induced by $H$. Then $\mathfrak{D}_{\mathfrak{P}^{\prime}}\left(H^{\prime}\right)$ is an affine polar space, that in itself is connected (cf. [4]). So there is in $\mathfrak{D}_{\mathfrak{P}^{\prime}}\left(H^{\prime}\right)$ a sequence of intersecting lines joining $K$ and $L$ as points of $\mathfrak{D}_{\mathfrak{P}^{\prime}}\left(H^{\prime}\right)$. However, lines of $\mathfrak{D}_{\mathfrak{P}^{\prime}}\left(H^{\prime}\right)$ are planes of $\mathfrak{D}_{\mathfrak{P}}(H)$. As $\mathcal{W} \subseteq H$ these planes are also planes of $\mathfrak{D}_{\mathfrak{P}}(\mathcal{W})$. 


\subsection{Parallelism}

Let $K_{1}, K_{2} \in \mathcal{L}_{\mathcal{W}}$. Then

$$
\begin{aligned}
K_{1} \|^{*} K_{2} \text { iff } \quad & K_{1} \cap K_{2}=\emptyset \text { and there are two distinct lines } \\
& L_{1}, L_{2} \in \mathcal{L}_{\mathcal{W}} \text { crossing both of } K_{1}, K_{2}, \text { such that } \\
& L_{1} \cap L_{2} \neq \emptyset \text { and } L_{1} \cap L_{2} \cap K_{i}=\emptyset \text { for } i=1,2 .
\end{aligned}
$$

In case there are are exactly 3 points per line in our polar space $\mathfrak{P}$, no two lines $K_{1}, K_{2}$ on an affine plane in $\mathfrak{D}_{\mathfrak{P}}(\mathcal{W})$ such that $K_{1} \|_{\mathcal{W}} K_{2}$ satisfy the right hand side of (3.1), as the required lines $L_{1}, L_{2}$ had to be of size 4 . This is why from now on we assume that

there are at least 4 points on every line of $\mathfrak{P}$.

Let $\|$ be the transitive closure of $\|^{*}$. It is clearly seen that $\| \subseteq \mathcal{L}^{*} \times \mathcal{L}^{*}$.

Lemma 3.4. The relation $\|$ is reflexive on $\mathcal{L}^{*}$.

Proof. Given a line $K_{1} \in \mathcal{L}_{\mathcal{W}}$, considering that the rank of $\mathfrak{P}$ is at least 3 , take a plane $\pi$ containing $K_{1}$ in a maximal singular subspace through $K_{1}$. There are lines $K_{2}, L_{1}, L_{2}$ on $\pi$ such that $K_{1} \cap K_{2}=\emptyset$ (that is $\left.K_{1}^{\infty}=K_{2}^{\infty}\right), L_{1} \neq L_{2}, L_{1} \cap L_{2} \neq \emptyset$, and $K_{i} \cap L_{j} \neq \emptyset$ for $i, j=1,2$. Thus $K_{1} \|^{*} K_{2}$ by (3.1). This means that $K_{1} \| K_{2}$ and $K_{2} \| K_{1}$, which by transitivity implies that $K_{1} \| K_{1}$.

Proposition 3.5. Let $\mathcal{W}$ be a subspace of $\mathfrak{P}$. The relation $\|_{\mathcal{W}}$ defined in (2.1) and the relation $\|$ coincide on the set of lines of $\mathfrak{D}_{\mathfrak{P}}(\mathcal{W})$.

Proof. Let $K_{1}, K_{2} \in \mathcal{L}_{\mathcal{W}}$. If $K_{1}=K_{2}$, then $K_{1} \|_{\mathcal{W}} K_{2}$ and $K_{1} \| K_{2}$. So, assume that $K_{1} \neq K_{2}$.

Consider the case where $K_{1} \|_{\mathcal{W}} K_{2}$. By (2.1) it means that $\bar{K} \cap \bar{L} \cap W \neq \emptyset$, and consequently $K_{1}^{\infty}=K_{2}^{\infty}=a$ for some $a \in \mathcal{W}$. This implies that $K_{1} \cap K_{2}=\emptyset$. Assume that $K_{1}$ and $K_{2}$ are coplanar, and $\Pi$ is the plane of $\mathfrak{D}_{\mathfrak{P}}(\mathcal{W})$ containing both of $K_{1}, K_{2}$. The plane $\bar{\Pi}$ is, up to an isomorphism, a projective plane, so it is Veblenian. Thus, by (3.1), $K_{1} \|^{*} K_{2}$. If $K_{1}$ and $K_{2}$ are not coplanar, then by Lemma 3.3 there is a sequence of planes $\Pi_{1}, \ldots, \Pi_{n}$ such that $K_{1} \subseteq \Pi_{1}, K_{2} \subseteq \Pi_{n}, a \in \overline{\Pi_{i}}$ for $i=1, \ldots, n$, and $\Pi_{j}, \Pi_{j+1}$ share a line for $j=1, \ldots, n-1$. Let $\Pi_{j} \cap \Pi_{j+1}=M_{j}$. Note that $a \in \overline{M_{1}}, \ldots, \overline{M_{n-1}}$ and $M_{j}, M_{j+1}$ are coplanar. Therefore $M_{j} \|^{*} M_{j+1}$. Moreover, $K_{1} \|^{*} M_{1}$ and $M_{n-1} \|^{*} K_{2}$ by the same reasons. So finally we get $K_{1} \| K_{2}$.

Now, assume that $K_{1} \|^{*} K_{2}$. Then $K_{1}, K_{2}$ are disjoint and coplanar. Thus $\overline{K_{1}}, \overline{K_{2}}$ meet in the closure of some plane, this means that they meet in $\mathcal{W}$. By (2.1) it gives $K_{1} \|_{\mathcal{W}} K_{2}$. If $K_{1} \| K_{2}$ then there is a sequence of proper lines $L_{1}, \ldots, L_{n}$ such that $K_{1}\left\|^{*} L_{1}\right\|^{*} \cdots\left\|^{*} L_{n}\right\|^{*} K_{2}$. So, from the previous reasoning we get $K_{1}\left\|\mathcal{W} L_{1}\right\| \mathcal{W}$ $\cdots\left\|_{\mathcal{W}} L_{n}\right\|_{\mathcal{W}} K_{2}$. As the relation $\|_{\mathcal{W}}$ is transitive we have $K_{1} \|_{\mathcal{W}} K_{2}$.

As an immediate consequence of Proposition 3.5 we get

Corollary 3.6. Affine lines can be distinguished in the set $\mathcal{L}_{\mathcal{W}}$ as those parallel to themselves. 


\subsection{Recovering}

If $\mathcal{W}$ is a hyperplane it follows by [4, Proposition 2.7] that:

Proposition 3.7. Let $\mathfrak{P}$ be a thick nondegenerate polar space of rank at least 2 and let $H$ be its hyperplane. The polar space $\mathfrak{P}$ can be recovered in the complement $\mathfrak{D}_{\mathfrak{P}}(H)$.

So, from now on we additionally assume that $\mathcal{W}$ is not a hyperplane. By Proposition 3.5 the relation $\|_{\mathcal{W}}$ can be expressed purely in terms of $\mathfrak{D}_{\mathfrak{P}}(\mathcal{W})$. Recall that our parallelism is partial: it is defined only on affine lines. However it is not a problem in view of Corollary 3.6. From Lemma 3.1(ii) there is a bijection between the sets $\mathcal{W}=\left\{L^{\infty}: L \in \mathcal{L}^{*}\right\}$ and $\left\{[L]_{\|}: L \in \mathcal{L}^{*}\right\}$. Thus we can recover $\mathcal{W}$ pointwise in a standard way:

points of the horizon $\mathcal{W}$ are identified with equivalence classes of parallelism i.e. directions of affine lines of the complement $\mathfrak{D}_{\mathfrak{P}}(\mathcal{W})$.

Let us introduce a relation $\sim \subseteq \mathcal{L}^{*} \times \mathcal{L}^{*}$ defined by the following condition:

$$
K_{1} \sim K_{2} \quad \text { iff } \quad \text { for all } M \in \mathcal{L}^{*} \text { if } M \cap K_{1} \neq \emptyset \text { then } M \nVdash K_{2} \text {. }
$$

In the sense of Euclid's Fifth Postulate it could be read as anti-euclidean parallelism. A lot more useful for us is its derivative $\equiv \subseteq \mathcal{L}^{*} /\left\|\times \mathcal{L}^{*} /\right\|$ defined as follows:

$$
\left[K_{1}\right]_{\|} \equiv\left[K_{2}\right]_{\|} \quad \text { iff } \quad \text { for all } M \in\left[K_{1}\right]_{\|}, N \in\left[K_{2}\right]_{\|}: M \sim N \text { and } N \sim M .
$$

Lemma 3.8. Let $M, N$ be two nonparallel affine lines. The following conditions are equivalent:

(i) $[M]_{\|} \equiv[N]_{\|}$,

(ii) there is a deep line $L \subseteq \mathcal{W}$, such that $M^{\infty}, N^{\infty} \in L$.

Proof. (i) $\Rightarrow$ (ii): From one-or-all axiom, $M^{\infty}$ must be collinear with at least one point of the line $\bar{N}$. Moreover, $M^{\infty}$ cannot be collinear with a proper point of $\bar{N}$, as $[M]_{\|} \equiv[N]_{\|}$. Thus $M^{\infty}$ is collinear with the unique improper point of $\bar{N}$, which is $N^{\infty}$.

Let $L$ be the line through $M^{\infty}, N^{\infty}$. Assume, that $\Pi$ is a semiaffine plane with $L=$ $\Pi^{\infty}$. Then, there are some affine lines $M_{1}, N_{1} \subseteq \Pi$ with $M^{\infty}=M_{1}^{\infty}$ and $N^{\infty}=N_{1}^{\infty}$. So, either $M_{1} \| N_{1}$ or $M_{1}$ and $N_{1}$ share a proper point. In view of (3.3), in both cases we get $[M]_{\|} \not \equiv[N]_{\|}$.

(ii) $\Rightarrow$ (i): Assume that $[M]_{\|} \not \equiv[N]_{\|}$. Due to (3.2) and (3.3) there is a proper point $a \in M$ and an affine line $K$ such that $a \in K \| N$ (or the symmetrical case holds). This means that $a$ and $N^{\infty}$ are collinear in $\mathfrak{P}$. The one-or-all axiom implies, that either there are no other points on $M$ that are collinear with $N^{\infty}$, or $N^{\infty}$ is collinear with all points on $M$. In the first case $N^{\infty}$ is not collinear with $M^{\infty}$, in the latter $\left\langle N^{\infty}, M\right\rangle \nsubseteq \mathcal{W}$ is the plane containing the line $\overline{M^{\infty}, N^{\infty}}$.

One can note, that the relation $\equiv$ defined by (3.3) and the relation $\equiv$ introduced in [4] coincide, though their definitions are expressed differently. Besides, our relation is not transitive, but the reflexive closure of its analogue in [4] is an equivalence relation. This benefit is the result of some hyperplane properties (see Lemma 3.1(i)). Nevertheless, we can overcome this inconvenience and define ternary relation of collinearity on the horizon $\mathcal{W}$. 
Lemma 3.9. If $K_{1}, K_{2}, K_{3}$ are pairwise nonparallel affine lines such that $\left[K_{i}\right]_{\|} \equiv$

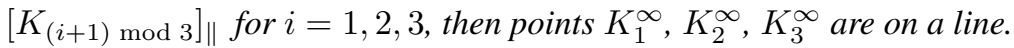

Proof. Let $a=K_{1}^{\infty}, b=K_{2}^{\infty}, c=K_{3}^{\infty}$. By Lemma 3.8 there are improper lines $L=\overline{a, b}$, $M=\overline{b, c}, N=\overline{c, a}$. Let $H$ be a hyperplane containing $\mathcal{W}$. If in $\mathfrak{D}_{\mathfrak{P}}(H)$ there is a plane, which closure contains one of the lines $L, M$ or $N$, then we also have such a plane in $\mathfrak{D}_{\mathfrak{P}}(\mathcal{W})$, that contradicts Lemma 3.8. Thus, $L, M, N \subseteq H$ are deep lines in relation to $\mathfrak{D}_{\mathfrak{P}}(H)$. By Lemma 2.3 of [4] this means that each of $L, M$ and $N$ contains a point of $\operatorname{rad} H$. Let $d \in \operatorname{rad} H$. Then, by Corollary 1.3 of [4], $H=d^{\perp},\{d\}=\operatorname{rad} H$, and $d$ is the unique deep point of $H$. As we have $d \in L, M, N$, it must be $L=M=N$.

Lemma 3.10. Let $K_{1}, K_{2}, K_{3}$ be pairwise nonparallel affine lines. Points $K_{1}^{\infty}, K_{2}^{\infty}, K_{3}^{\infty}$ are on a line iff one of the following holds:

(i) there are affine lines $M_{1}\left\|K_{1}, M_{2}\right\| K_{2}, M_{3} \| K_{3}$ such that $M_{1}, M_{2}, M_{3}$ form a triangle in $\mathfrak{D}_{\mathfrak{P}}(\mathcal{W})$,

(ii) $\left[K_{1}\right]_{\|} \equiv\left[K_{2}\right]_{\|},\left[K_{2}\right]_{\|} \equiv\left[K_{3}\right]_{\|}$, and $\left[K_{3}\right]_{\|} \equiv\left[K_{1}\right]_{\|}$.

Proof. Assume that $K_{1}^{\infty}, K_{2}^{\infty}, K_{3}^{\infty}$ are on a line $L$. If (i) does not hold, then there is no plane $\Pi$ in $\mathfrak{D}_{\mathfrak{P}}(\mathcal{W})$ with $L=\Pi^{\infty}$. This means that $L$ is a deep line and by Lemma 3.8 we get (ii).

Now, assume that (i) is the case. Take a plane $\Pi$ spanned by the triangle $M_{1}, M_{2}, M_{3}$. Then $K_{1}, K_{2}, K_{3} \subseteq \Pi$ and $K_{1}^{\infty}, K_{2}^{\infty}, K_{3}^{\infty}$ are on a line $\Pi^{\infty}$. If (ii) is fulfilled then $K_{1}^{\infty}$, $K_{2}^{\infty}, K_{3}^{\infty}$ are on a line directly by Lemma 3.9.

The meaning of Lemma 3.10 is that we are able to recover improper lines regardless of whether $\mathcal{W}$ is flappy or not. Let $\left[[K]_{\|},[L]_{\|}\right]_{\equiv}:=\left\{[M]_{\|}:[M]_{\|} \equiv[K]_{\|},[L]_{\|}\right\}$. Then new lines can be grouped into two sets:

$$
\begin{aligned}
\mathcal{L}^{\prime}: & =\left\{\left[[K]_{\|},[L]_{\|}\right]_{\equiv}:[K]_{\|} \equiv[L]_{\|} \text {and } K \nVdash L\right\}, \\
\mathcal{L}^{\prime \prime}: & =\left\{\Pi^{\infty}: \Pi \text { is a semiaffine plane of } \mathfrak{D}_{\mathfrak{P}}(\mathcal{W})\right\} .
\end{aligned}
$$

All our efforts in this paper essentially amount to the following isomorphism

$$
\mathfrak{P} \cong\left\langle S_{\mathcal{W}} \cup \mathcal{L}^{*} / \|, \mathcal{L}_{\mathcal{W}} \cup \mathcal{L}^{\prime} \cup \mathcal{L}^{\prime \prime}, \mid\right\rangle
$$

A new point $[K]_{\|}$is incident to a line $L \in \mathcal{L}_{\mathcal{W}}$ iff $K \| L$. It is incident to a line $L \in \mathcal{L}^{\prime}$ iff there is $M \in \mathcal{L}_{\mathcal{W}}$ such that $\left[[K]_{\|},[M]_{\|}\right]_{\equiv}=L$. Eventually, it is incident to a line $L \in \mathcal{L}^{\prime \prime}$ iff $K \subseteq \Pi$ and $L=\Pi^{\infty}$.

Theorem 3.11. Let $\mathfrak{P}$ be a nondegenerate, embeddable polar space of rank at least 3 , with at least 4 points per line, and $\mathcal{W}$ be its subspace, that is contained in a hyperplane. The polar space $\mathfrak{P}$ can be recovered in the complement $\mathfrak{D}_{\mathfrak{P}}(\mathcal{W})$.

\section{References}

[1] J. Bamberg, J. De Beule and F. Ihringer, New non-existence proofs for ovoids of Hermitian polar spaces and hyperbolic quadrics, Ann. Comb. 21 (2017), 25-42, doi:10.1007/ s00026-017-0346-0. 
[2] P. J. Cameron, Projective and Polar Spaces, volume 13 of QMW Maths Notes, Queen Mary and Westfield College, School of Mathematical Sciences, London, 1992, http://www . maths . qmul.ac.uk/ pjc/pps/.

[3] A. M. Cohen, Point-line spaces related to buildings, in: F. Buekenhout (ed.), Handbook of Incidence Geometry: Buildings and Foundations, North-Holland, Amsterdam, pp. 647-737, 1995, doi:10.1016/b978-044488355-1/50014-1.

[4] A. M. Cohen and E. E. Shult, Affine polar spaces, Geom. Dedicata 35 (1990), 43-76, doi: $10.1007 / \mathrm{bf00147339.}$

[5] H. Karzel and H. Meissner, Geschlitze Inzidenzgruppen und normale Fastmoduln, Abh. Math. Sem. Univ. Hamburg 31 (1967), 69-88, doi:10.1007/bf02992387.

[6] H. Karzel and I. Pieper, Bericht über geschlitzte Inzidenzgruppen, Jber. Deutsch. Math. Verein. 72 (1970), 70-114, http: / / eudml.org/doc/146588.

[7] A. Kreuzer, Semiaffine spaces, J. Comb. Theory Ser. A 64 (1993), 63-78, doi:10.1016/ 0097-3165(93)90088-p.

[8] M. Marchi and S. Pianta, Partial parallelism spaces and slit spaces, in: A. Barlotti, P. V. Ceccherini and G. Tallini (eds.), Combinatorics '81, North-Holland, Amsterdam-New York, volume 18 of Annals of Discrete Mathematics, 1983 pp. 591-600, doi:10.1016/s0304-0208(08) 73337-1, proceedings of the International Conference on Combinatorial Geometries and their Applications held in Rome, June 7 - 12, 1981.

[9] K. Petelczyc and M. Żynel, The complement of a point subset in a projective space and a Grassmann space, J. Appl. Logic 13 (2015), 169-187, doi:10.1016/j.jal.2015.02.002.

[10] K. Petelczyc and M. Żynel, Affinization of Segre products of partial linear spaces, Bull. Iranian Math. Soc. 43 (2017), 1101-1126, http: / / bims.iranjournals.ir/article_ 1010. html. 